\title{
Atmospheric Studies with the Tri-Band Beacon Instrument on the COSMIC Constellation
}

\author{
Paul A. Bernhardt ${ }^{1, *}$, Craig A. Selcher ${ }^{2}$, Santi Basu ${ }^{3}$, \\ Gary Bust ${ }^{4}$ and Steven C. Reising ${ }^{5}$
}

(Manuscript received 30 September 1999, in final form 8 November 1999)

\begin{abstract}
Radio frequency transmissions from each satellite in the Constellation Observing System for Meteorology, Ionosphere, and Climate (COSMIC) can be used to study the plasma in the upper atmosphere by (1) providing profiles and two dimensional images of the ionosphere and (2) monitoring phase and amplitude scintillations induced in radio waves propagating through the ionosphere. In addition, the received radio data can be applied to the neutral atmosphere by (a) detecting horizontal fluctuations in tropospheric water vapor and (b) yielding accurate position data for satellite drag and neutral density determination. A three-frequency radio beacon called the Tri-Band Beacon (TBB) is being developed for the COSMIC program to provide transmissions at VHF, UHF and L-band. Tomographic imaging of the ionosphere is a recently developed technique that uses integrated measurements and computer reconstructions to determine electron densities. The integral of electron density along vertical or oblique paths is obtained by employing radio transmissions from low-earth-orbiting (LEO) COSMIC satellite transmitters to a chain of receivers on the earth's surface. Analyzing the total electron content (TEC) data using computerized ionospheric tomography (CIT) produces two-dimensional maps of the ionospheric plasma.Difficulties associated with CIT arise from the non-uniqueness of the reconstructions owing to limited angle measurements or nonoptimal receiver. Improvements in both reconstruction algorithms and CIT measurement systems are being implemented for the COSMIC mission by combining the GPS occultation data with the TBB measurements of TEC.
\end{abstract}

\footnotetext{
${ }^{1}$ Beam Physics Branch, Plasma Physics Division, Naval Research Laboratory, Washington, DC USA

${ }^{2}$ Transmission Technology Branch, Information Technology Division, Naval Research Laboratory, Washington, DC USA

${ }^{3}$ Air Force Geophysics Laboratory, Hanscom AFB, MA, USA

${ }^{4}$ Applied Research Laboratory, University of Texas at Austin, Austin, TX, USA

${ }^{5}$ Microwave Remote Sensing Laboratory, University of Massachusetts., Amherst, MA, USA

* Corresponding author address: Dr. Paul A. Bernhardt, Code 6794, Naval Research Laboratory, Washington, DC 20375 USA; E-mail: bern@ppdu.nrl.navy.mil
} 
Once the ionospheric effects on the radio wave propagation have been determined, the Doppler shifts of the UHF and VHF transmissions can be analyzed to give positioning of the COSMIC satellites to within a few meters. By measuring the effects of the atmospheric drag on the COSMIC satellites, global data for neutral densities in the upper atmosphere can be obtained. There is an additional phase delay of the VHF/UHF/L-bands from the neutral constituents of the troposphere. This phase delay can be used to provide measurements of integrated water vapor. The spatial distribution of water vapor density may be determined with high precision phase measurements from a linear array of ground receivers observing the $L$-band transmissions of the COSMIC TBB.

(Key words: Electron density, Tomography, Radio scintillations, Ionosphere, Radio beacon)

\section{SCIENTIFIC OBJECTIVES}

The Constellation Observing System for Meteorology, Ionosphere, and Climate (COSMIC) uses multi-point sensors that employ line-of-sight measurements for studying the Earth's atmosphere. The three sensors on each COSMIC satellite are (1) the Tri-Band Beacon (TBB), (2) Tiny Ionospheric Photometer (TIP), and (3) GPS occultation receiver. These instruments are complementary in their observations by using both radio and optical measurements. The eight COSMIC satellites will be placed in planes separated by 22.5 degrees at $800-\mathrm{km}$ operational altitude and 72 degrees inclination. The COSMIC system has been described in detail by Rocken et al. (2000). The Tri-Band Beacon (TBB) radiates phase coherent signals to receivers for studies of both the upper atmosphere and the troposphere.

The primary objective of the Tri-Band Beacon experiment on COSMIC is to study the electron density in the Earth's ionosphere. This is analyzing total electron content (TEC) data to produce electron densities as either two-dimensional maps or one-dimensional profiles. The earth's upper atmosphere contains a partially ionized plasma that is constantly changing under the influence of solar extreme ultraviolet (EUV) radiation, recombination chemistry, neutral winds, and electric fields (Rishbeth and Garriott, 1969; Kelley, 1989). The ionosphere extends from $50 \mathrm{~km}$ to above 1000-km altitude with variations in ion species balanced by equal densities of electrons. At altitudes below $150 \mathrm{~km}$, the ions are primarily molecular. The peak densities are found in the F-layer near 300 to $400 \mathrm{~km}$ where atomic oxygen is the primary ion species. Above 1500-km altitude, the plasmasphere is composed of atomic hydrogen and helium ions along with an equal number of electrons to maintain neutrality. The atmospheric region known as the ionosphere is both important and complex. The ionosphere affects terrestrial radio signals. Satellite to ground links are affected by electron density irregularities that can degrade received signal strength. Communication, radar, and navigation systems often rely on predictions and measurements of ionospheric propagation conditions. Over-the-horizon radars require accurate models of the ionosphere to determine target locations. 
The second objective of the Tri-Band Beacon is to measure the influence of ionospheric irregularities on VHF (150 MHz), UHF (400 MHz) and L-Band (1067 MHz) transmissions from space to the ground. This is accomplished by recording amplitude and phase scintillations between the COSMIC beacon and ground receivers. The ionosphere of ten becomes disturbed under the influence of electric fields, neutral winds, and gravity. These forces distort the plasma structures to form irregularities aligned with the earth's magnetic field. When the irregularity scale sizes are on the order of a Fresnel zone size, which is about $1 \mathrm{~km}$ for UHF signals from satellites, the waves on the ground become distorted and undergo large fluctuations or "scintillations" in the amplitude and phase. The sources of irregularities vary with latitude. The high latitude ionosphere is coupled to the solar wind via magnetic field lines. The earth's magnetosphere becomes charged by the solar wind. Ionospheric irregularities called patches are formed by a gradient-drift instability driven by high-latitude convection. Also at high latitudes, field-aligned currents in the F-region and horizontal currents in the Eregion are linked to the solar wind and magnetosphere. All of these high latitude phenomena are dependent on the magnetic activity, interplanetary magnetic field (IMF) and season. On the other hand, the low-latitude ionosphere becomes disturbed because of gravity and driftdriven instabilities. At low latitudes, the magnetic field lines are nearly horizontal and gravity operates perpendicular to B. A gxB force produces electric field polarization of the plasma on the bottomside of the equatorial F-layer. The layer can be affected by a Rayleigh-Taylor type instability and large "bubbles" can form and rise through the F-layer to the topside ionosphere. The internal instabilities operate at night to form large areas of ionospheric irregularities. One goal of the TRI-Band Beacon on COSMIC is to provide a database for understanding these instability processes and determine their influence on radio propagation.

The third application of the TBB is to monitor tropospheric water vapor with the lowearth orbiting Doppler beacons. The observation of carrier phase of one or more COSMIC beacons at several receiving sites provides slant water vapor delays. The water vapor "wet" phase delay is measurable if the effects of ionospheric delays, orbit position, dry atmospheric delays, and oscillator drifts can be removed with sufficient accuracy. The dry gases and water vapor in the neutral atmosphere refract radio waves to yield delays from the troposphere. The dry component of the delay is predictable from surface measurements and is far less variable than the "wet" component due to water vapor. The wet delay is a simple function of the precipitable water vapor (PWV) along the line-of-sight between the receiver and transmitter. PWV is a highly variable atmospheric constituent. This use of the radio beacon can provide a source of water vapor measurements that are relatively inexpensive compared to measurements from radiometers. The orbit geometry of the LEO COSMIC satellites makes the measurements from the TBB well suited for the use of tomographic techniques. Two-dimensional images of the water vapor in a plane containing the satellite orbit can be obtained using a chain of ground receivers separated by $1 \mathrm{~km}$ or less. The main advantage of TBB water vapor measurements over GPS measurements of tropospheric water vapor (Bevis et al., 1992; Bevis et al., 1994; Rocken et al., 1997) is that the TBB signals come from rapidly-moving, lowearth-orbit satellites that provide much more rapid two-dimensional slices of the atmosphere than are provided by the slower orbiting GPS satellites. The rapid scan of the troposphere can provide information on horizontal gradients that enables the production of a 2-D tomographic 
image. The TBB experiment on COSMIC is designed to provide images of the distribution of water vapor which can be applied to atmospheric research and improved weather forecasting.

The fourth use of the COSMIC beacon is to provide accurate determination of the COSMIC satellite position using Doppler tracking. By measuring the Doppler shift of the VHF, UHF and L-Band signals relative to a reference frequency at receivers on the ground, one can deduce the range and relative velocity of the satellite. Two signals at different frequencies are used to correct for the effects of ionospheric refraction on the propagation of the signal. Precisely time tagged measurements of the Doppler frequency at three accurately positioned ground receivers simultaneously observing the TBB transmissions are required to compute the satellite orbit parameters. The Doppler technique has been used since 1975 with the GEOSC(1975-1980), GEOSAT(1985-1990), RADCAL(1993-present) and GFO(1997-present) satellites to provide position accuracy of 3 to 5 meters (A. Limon, Personal Communication, 1999). Atmospheric drag is the largest and least well characterized force that perturbs the orbit of the COSMIC satellites in the upper atmosphere. Time-dependent global corrections to satellite drag models can be derived based on assimilation of Doppler ranging data obtained with the COSMIC TBB.

\section{MEASUREMENT TECHNIQUE}

All of the measurements from the COSMIC TBB rely on measurements of the variations in the phase of radio waves propagating from the beacon to ground-based receivers. These variations are produced by fluctuations in the refractive index of the earth's atmosphere and by the motion of the spacecraft. The radio wave refractive index is given by

$$
\mathrm{n}=1+10^{-6}\left(\mathrm{~N}_{\mathrm{dry}}+\mathrm{N}_{\text {wet }}\right)-\frac{X}{2} \mathrm{~m} \frac{X Y_{\mathrm{L}}}{2}
$$

where $\mathrm{N}$ is refractivity $\mathrm{N}_{\text {dry }}=77.64 \mathrm{P}_{\text {dry }} / \mathrm{T}, \mathrm{N}_{\text {wet }}=71.7 \mathrm{P}_{\text {wet }} / \mathrm{T}+10^{5} 3.744 \mathrm{P}_{\text {wet }} / \mathrm{T}^{2}, \mathrm{P}_{\text {dry }}$ is dry-air pressure in $\mathrm{mBar}$ or $\mathrm{hPa}, \mathrm{P}_{\text {wet }}$ is the water-vapor pressure, $\mathrm{T}$ is the temperature in Kelvin, $\mathrm{X}=\mathrm{N}_{\mathrm{e}} \mathrm{e}^{2} /\left(\varepsilon_{0} \mathrm{~m}_{\mathrm{e}} \omega^{2}\right) \mathrm{Y}_{\mathrm{L}}=\mathrm{eB}_{\mathrm{L}} /\left(\mathrm{m}_{\mathrm{e}} \omega\right), \mathrm{N}_{\mathrm{e}}$ is the electron density in $\mathrm{m}^{-3}$, e is electron charge, $\varepsilon_{0}$ is the free space permittivity, $m_{e}$ is the electron mass, $B_{L}$ is the component of the earth's magnetic field along the direction of propagation, and $\omega=2 \pi f$ is the wave frequency in radians/second. The first three terms are dependent on temperature and the effects of tropospheric variations in density and water-vapor partial pressure. The last two terms in (1) are the ionospheric contribution, which includes the influence of electron density and magnetic fields through the magnetoionic parameters $X$ and $Y_{L}$, respectively. The ionospheric term is derived with the simplifying assumption that the waves do not propagate perpendicular to the magnetic field direction. The - and + signs in the last term of (1) denote the ordinary and extraordinary modes of polarization in the ionosphere.

It has been demonstrated that ionospheric density variations can be reconstructed from total electron content (TEC) data obtained from orbiting satellites (Austen, Franke and Liu, 1988; Raymund et al., 1990; Sayenko et al., 1991). Over the past 10 years, groups in Europe (Kersley et al., 1997; Fehmers 1996), Asia (Yeh et al., 1994; Huang et al., 1999), United States (Bust et al., 1994), Russia (Kunitsyn et al., 1994) and Russia/America jointly (Foster et al., 
1994) have carried out studies of the ionosphere using CIT.

Five different techniques are available to measure electron content using satellite beacons (Yeh and Raymund, 1991). With the COSMIC TBB experiment, the electron content will be determined from the differential phase of a pair of continuous wave signals transmitted by the Tri-Band Beacon at phase-related frequencies $\left(f_{1}\right.$ and $f_{2}$ ). If the frequencies are much higher than the maximum plasma frequency $\left(f_{p}\right)$ in the ionosphere, then refractive bending and magnetic field effects can be neglected along the propagation path. With these assumptions, the received phase from a satellite at distance $D$ is derived using (1) to give

$$
\Phi(\mathrm{f})=\frac{2 \pi \mathrm{fD}}{\mathrm{c}}+\frac{2 \pi \mathrm{f}}{\mathrm{c}} \int\left(\mathrm{N}_{\mathrm{dry}}+\mathrm{N}_{\text {wet }}\right) \mathrm{ds}-\frac{\pi \kappa}{\mathrm{fc}} \int \mathrm{n}_{\mathrm{e}} \mathrm{ds} \text { where } \kappa=\left(\frac{\mathrm{e}}{2 \pi}\right)^{2} \frac{1}{\mathrm{~m}_{\mathrm{e}} \varepsilon_{0}} .
$$

Highly stable reference oscillators at both transmitter and receiver permit direct observations of the phase changes. The ionospheric contribution, however, can be determined by using the differential phase between two frequencies because of frequency dependence in (2). Scaling the two phases by the ratio of the two frequencies yields a measure of the integrated electron density along the propagation path according to:

$$
\mathrm{C} \int_{\mathrm{R}}^{\mathrm{T}} \mathrm{n}_{\mathrm{e}} \mathrm{ds}=\Phi_{1}-\Phi_{2} / \mathrm{N}+\Psi \text { where } \mathrm{N}=\mathrm{f}_{2} / \mathrm{f}_{1} .
$$

For $\mathrm{f}_{1}=150 \mathrm{MHz}$ and $\mathrm{f}_{2}=400 \mathrm{MHz}$, the constant $\mathrm{C}=5.62 \times 10^{-15}$ radians $-\mathrm{m}^{2}$ and a differential phase measurement precision of 0.1 radians yields a TEC accuracy of $1.9 \times 10^{13} \mathrm{~m}^{-2}$. This represents a resolution of one part in $10^{3}$ in the typical ionosphere. For the other pair of frequencies, $\mathrm{f}_{2}=400 \mathrm{MHz}$ and $\mathrm{f}_{3}=10662 / 3 \mathrm{MHz}$, a differential phase measurement of 0.1 radian yields a TEC accuracy of $5.1 \times 10^{13} \mathrm{~m}^{-2}$. The resolution of TEC measurements is determined by the lower frequency of transmission. Consequently, the $150 / 400 \mathrm{MHz}$ pair provides the best resolution but has the greatest susceptibility to degradation as the waves propagate through ionospheric irregularities. The three frequencies for the Tri-Band Beacon have been chosen to provide optimum TEC sensitivity and resistance to scintillations induced by ionospheric irregularities. Absolute TEC often cannot be measured with the differential phase technique because of multiples of $2 \pi$ in the phase measurement. Multiple frequencies can be used to resolve the $2 \pi$ phase ambiguities.

Once the ionospheric phase enhancement is determined, it can be eliminated from (2). The dry vapor pressure can be accurately measured and modeled to remove the dry refractivity $\left(\mathrm{N}_{\mathrm{dry}}\right)$ from (2). The remaining terms derived from the phase are the slant wet phase delay

$$
\Phi_{\text {wet }}(f)=\frac{2 \pi f}{c} \int N_{\text {wet }} \text { ds, }
$$

and the Doppler frequency shift defined as

$$
2 \pi \mathrm{f}_{\mathrm{D}}=\frac{\partial}{\partial \mathrm{t}} \Phi(\mathrm{f})=\frac{2 \pi \mathrm{f}}{\mathrm{c}} \frac{\partial \mathrm{D}}{\partial \mathrm{t}}+\frac{2 \pi \mathrm{f}}{\mathrm{c}} \frac{\partial}{\partial \mathrm{t}} \Phi_{\text {wet }} .
$$

The two contributions in (5) can be separated by examining their two different time scales. The hystrostatic delay fluctuations (4) are expected to have time scales of a few minutes as the 
satellite passes over a region of localized water vapor. The description of the satellite trajectory, on the other hand, results from long term monitoring of the Doppler frequency fD over a period of one orbit. Precise orbit determination by analysis of the TBB Doppler frequencies will be compared to and will complement the position data provided by the GPS receiver on COSMIC. This direct analysis of the received phase is only useful if the drift between the transmitted frequency and receiver reference is negligible. The COSMIC TBB has been designed to provide a frequency stability of 1 part in $10^{12}$ (per 15 minute duration of a pass) using an oven-stabilized oscillator. Similar stability has been used on previous satellites such as RADCAL to provide position accuracy of better than 5 meters.

Rapid fluctuations in radio wave phase occur from diffraction of the radio wave after propagation through a region of the ionosphere that contains irregularities in electron density. These phase fluctuations cannot be simply related to the integral of simple quantities such as those given by (2). Instead, scintillations are treated in terms of statistical fluctuations. While passing through ionospheric irregularities, radio waves from satellites undergo phase fluctuations as given by (2). As the wave emerges from the irregularity and propagates to the ground, these phase fluctuations interfere and a diffraction pattern in both intensity and phase develops on the ground. In the presence of a relative motion between the satellite, the ionosphere and/ or the receiver, rapid fluctuations of signal intensity and phase, known as scintillations, are recorded. The magnitude of the intensity scintillation is characterized by the variance in the received power fluctuations where the $\mathrm{S}_{4}$ scintillation index is defined as the square root of the variance of received power fluctuations divided by the mean value of the received power

$$
\mathrm{S}_{4}=\frac{\sqrt{\left\langle\mathrm{P}^{2}\right\rangle-\langle\mathrm{P}\rangle^{2}}}{\langle\mathrm{P}\rangle},
$$

where $<\mathrm{P}>$ denote the time-averaged power. The magnitude of the scintillation index typically decreases with increasing frequency because (a) the initial phase perturbation is inversely proportional to the wave frequency as given by (2) and (b) the irregularity power spectrum has a power law fall off. Similar behavior is seen with the phase scintillation index, $\sigma_{\phi}$ which is defined as the standard deviation of phase $(\phi)$ over a specific interval of time

$$
\sigma_{\phi}=\sqrt{\left\langle\phi^{2}\right\rangle-\langle\phi\rangle^{2}},
$$

where the average is typically computed over 10 seconds for an orbiting satellite. The TriBand Beacon provides the capability of measuring both types of radio wave scintillations at each of its VHF, UHF, and L-band frequencies.

\section{INSTRUMENT DESCRIPTION}

Each of the TBB measurement techniques requires high stability and phase coherence for the three transmitted frequencies. A block diagram of the scheme for frequency generation on the Tri-Band Beacon (TBB) is illustrated in Fig. 1. The three carrier frequencies $(150.012$, 400.032 and $1066.752 \mathrm{MHz}$ ) are derived from a stabilized crystal oscillator operating at 16.668 MHz. This "ovenized" oscillator has an inherent stability of 1 part in $10^{12}$ per 15 -minute pass. 


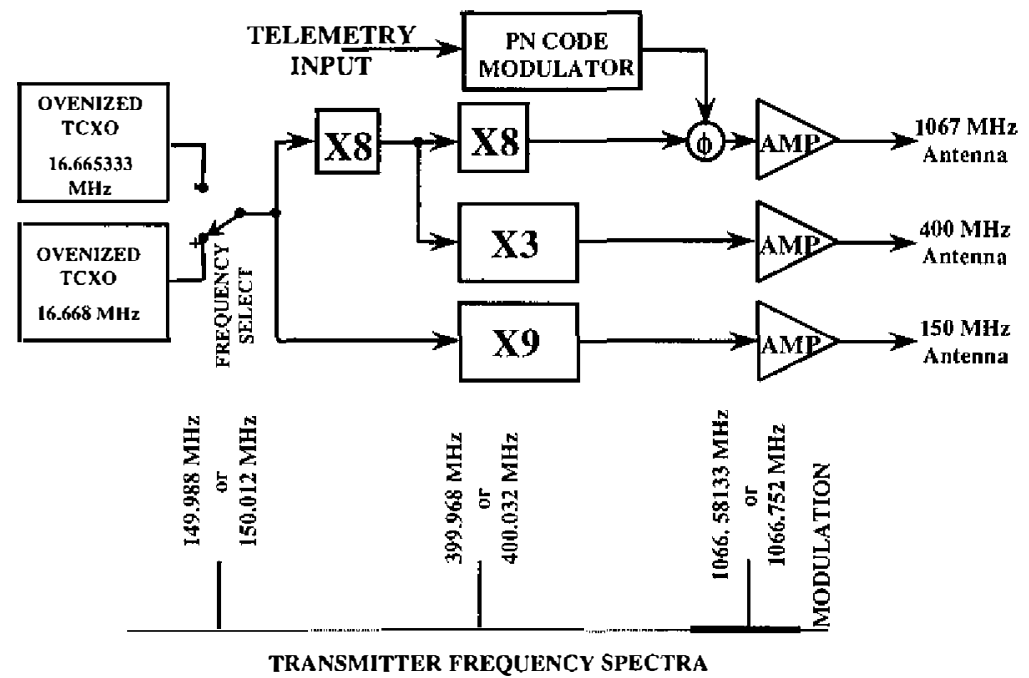

Fig. l. Tri-Band Beacon for use as a differential phase tomography transmitter. The RF spectra show monochromatic carriers from coherent radio frequency generation and low-level broad band modulation by a pseudonoise (PN) code.

By switching to the $16.665333 \mathrm{MHz}$ reference, another set of frequencies $(149.988,399.968$, and $1066.58133 \mathrm{MHz}$ ) can be selected to accommodate existing receivers that are permanently tuned to the TRANSIT satellite frequencies. The L-band carrier near $1067 \mathrm{MHz}$ is phase-modulated to send spacecraft data to ground telemetry receivers. The modulation scheme can be turned off when the L-band carrier is used for differential phase measurements. The TBB design is based on beacons previously integrated into the DMSP/S15 and STRV-1d spacecraft.

The antenna for the TBB was designed to preserve the relative phase among the three frequencies (Fig. 2). The VHF and UHF signals are transmitted with crossed dipoles that are fed in quadrature to yield circular polarization. Each dipole has a $400 \mathrm{MHz}$ trap to make it dual frequency. The third L-band frequency is radiated from a quadrifilar helix. All three antennae provide circular polarization below and horizontal polarization at the sides of each COSMIC satellite. The transmitter power of the TBB and the gain of the antenna must work in concert to provide at least $-140 \mathrm{dBm}\left(10^{-17}\right.$ Watts $)$ with a uniform phase front at the receiver. Table 1 lists the system requirements for satellite to ground links in both a quiescent and a turbulent ionosphere.

\section{ELECTRON DENSITY PROFILES (EDP) FROM THE OVERFLIGHT OF A SINGLE RECEIVER}

The simplest model of TBB operation involves one ground station continuously recording the amplitudes of transmissions from a single COSMIC satellite at 150,400 and 1067 


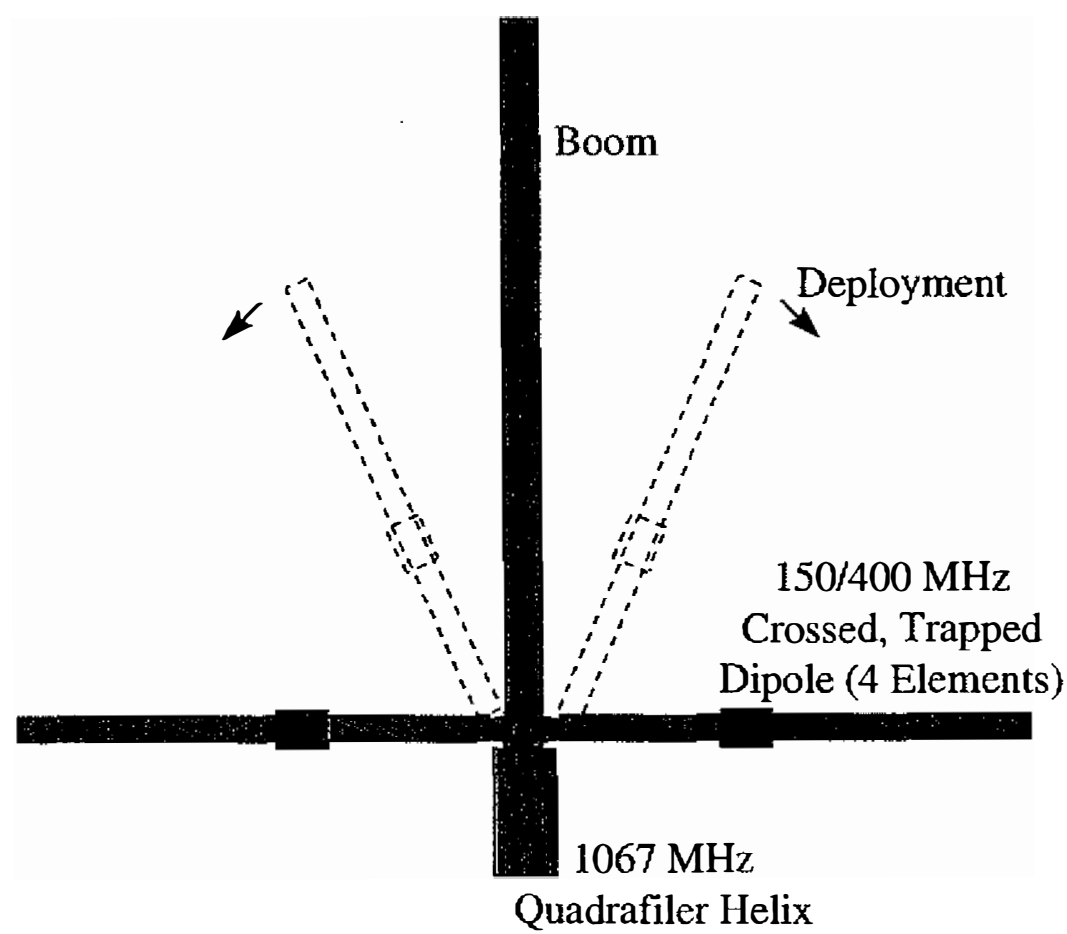

Fig. 2. Three frequency antennae deployed from each COSMIC spacecraft. The antennae provide circularly polarized transmissions with a common phase center.

Table 1. Tri-Band Beacon Link Budget

"Values for a turbulent ionosphere

\begin{tabular}{llll}
\hline SUBJECT & $150 \mathrm{MHz}$ & $400 \mathrm{MHz}$ & $1067 \mathrm{MHz}$ \\
\hline Transmitter Power, dBm & $27(0.5 \mathrm{~W})$ & $30(1.0 \mathrm{~W})$ & $33(2.0 \mathrm{~W})$ \\
Cable Loss, dB & -1 & -2 & -3 \\
TX Antenna Gain, dB & 2 & 2 & 2 \\
Path Loss, dB (10 Elev.) & -143.5 & -152 & -160.5 \\
Scintillation, dB & $0(-20)^{*}$ & $0(-8)^{*}$ & $0(-2)^{*}$ \\
Polarization Loss, dB & 0 & 0 & 0 \\
RX Antenna Gain, dB & 2 & 2.5 & 3.5 \\
\cline { 2 - 4 } Signal At Receiver, dBm & $-113.5(-133.5)^{*}$ & $-119.5(-127.5)^{*}$ & $-122(-124)^{*}$ \\
Required Signal, dBm & -130 & -130 & -130 \\
\cline { 2 - 4 } Margin, dBm & $16.5(-3.5)^{*}$ & 10.5 & $8(6)^{*}$ \\
\hline
\end{tabular}


MHz. The outputs of phase detectors in the receiver are scaled according to (3) to yield a continuous record of total electron content from horizon to zenith to horizon as the COSMIC beacon crosses over the ground station. A sample of this type of data was obtained during an over flight that occurred using the two-frequency radio beacon on the ARGOS satellite (Fig. 3).

Deriving a profile from the TEC data in Fig. $3 \mathrm{c}$ is based on an assumption that the ionosphere is spherically stratified into shells and depends only on altitude (z) with the following functional form

$$
\mathrm{N}_{\mathrm{c}}^{(\mathrm{H})}(\mathrm{z})=\mathrm{N}_{\max } \exp \left(\frac{1-\mathrm{H}^{\prime}\left(\mathrm{z}^{\prime}\right)-\mathrm{e}^{-\mathrm{H}^{\prime}\left(\mathrm{z}^{\prime}\right)}}{2}\right)
$$

where $\mathrm{N}_{\text {nax }}=$ density at the peak, $\mathrm{H}^{\prime}=\mathrm{z}^{\prime} / \mathrm{H}, \mathrm{H}=\mathrm{H}_{0}+\mathrm{H}_{1} \mathrm{z}^{\prime}+\mathrm{H}_{2} \mathrm{z}^{\prime 2}, \mathrm{z}^{\prime}=\mathrm{z}-\mathrm{z}_{\max }$, and $\mathrm{z}_{\max }=$ altitude of the layer peak. Equation (8) called a "Chapman Layer" is a convenient functional form having the approximate shape of the F-layer density profile and has five parameters $\mathrm{H}_{0}$, $\mathrm{H}_{1}, \mathrm{H}_{2}, \mathrm{~N}_{\max }$, and $\mathrm{z}_{\max }$, which can be adjusted to fit the TEC data through a line-of-sight integration through the profile in curved earth geometry. The shape of the layer is extracted from the slant TEC data because the low elevation observations have larger weighting toward lower altitudes. The nearly vertical TEC data have equal weighting of the electron data at all altitudes. The slant TEC is defined as

$$
\mathrm{I}(\theta)=\int_{\mathrm{s}(\theta)} \mathrm{N}_{\mathrm{e}}(\mathrm{z}, \theta, \phi) \mathrm{ds}
$$

where the integration path $S(\theta)$ is straight line between the satellite and receiver, $\phi$ is an angle perpendicular to the satellite orbit, and $\theta$ is the geocentric angle along the satellite orbit. This angle is taken to be zero at the point of closest approach for the satellite. For polar orbits, $\theta$ can be taken to be the latitude of the satellite relative to the receiver latitude and $\phi$ can be longitude relative to the receiver. If the ionosphere were spherically stratified, then $I(\theta)$ would be an even function. In the case shown in Fig. 3c, the profile estimation is performed on the even part of the TEC ata, and the odd part is used to estimate the ionospheric density gradient. The even and odd parts are

$$
I_{E}(\theta)=\frac{I(\theta)+I(-\theta)}{2} \text { and } I_{O}(\theta)=\frac{I(\theta)-I(-\theta)}{2}
$$

where $\mathrm{I}(\theta)=\mathrm{I}_{\mathrm{E}}(\theta)+\mathrm{I}_{\bullet}(\theta)$.

The profiling procedure fits the Chapman Layer function (8) using the even part of the TEC measurements. The horizontal density gradients are estimated using the odd part of the TEC data. Neglecting any electron density variations in the $\phi$-direction, the electron density function is written as

$$
\mathrm{N}_{\mathrm{e}}(\mathrm{z}, \theta)=\mathrm{N}_{\mathrm{e}}^{(\mathrm{H})}(\mathrm{z})[1+\mathrm{P}(\theta)]
$$

where all variations along the satellite path are taken to be in peak density variations for a fixed profile shape. Substitution of (11) into (9) leads to the solutions 

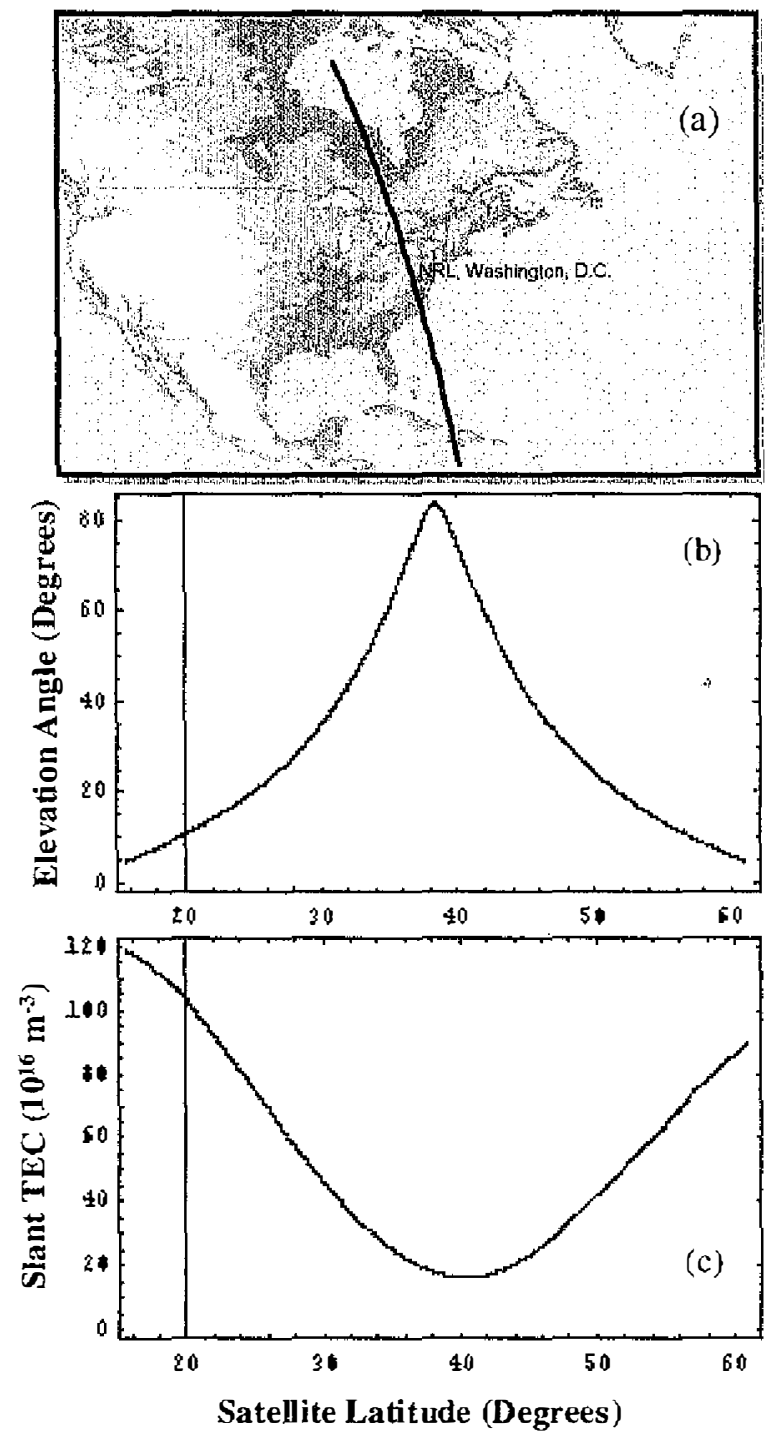

Fig. 3. ARGOS/CERTO observations at 1847 UT on 28 February 1999. The ARGOS ground track (a) permitted observations of the two-frequency CERTO beacon operating at 150.012 and $400.032 \mathrm{MHz}$ using a TEC receiver at the National Research Laboratory. The satellite elevation reached a maximum of 83 degrees (b) over the receiving side. The TEC data (c) showed no irregularities but the asymmetry of the curve indicated a denser ionosphere to the south of the receiver.

$$
I_{E}(\theta)=\int_{s(\theta)} N_{e}^{(H)}(z) d s \text { and } I_{o}(\theta)=\int_{s(\theta)} N_{e}^{(H)}(z) P(\theta) d s=I_{E}(\theta) P(\theta)
$$

Solving the second equation in (11) for $P(\theta)$ and substitution into (10) yields a two-dimensional representation of the ionosphere that is consistent with the single station TEC measurements. This function can be used to describe the ionosphere and its horizontal gradients near the closest point of approach for the satellite. Model studies have shown that this procedure provides highly accurate measurements of vertical TEC and $\mathrm{N}_{\max }$ of the F-layer to within a few percent. The estimates of peak density $\left(\mathrm{N}_{\max }\right)$ and layer thickness $\left(\mathrm{H}_{0}\right)$ can be off by as much as $20 \%$ because an overestimate in peak density can be compensated by an estimate under in thickness to yield the same value of TEC. 


\section{IONOSPHERIC IMAGES FROM LINEAR ARRAYS OF GROUND-BASED RE- CEIVERS}

The COSMIC TBB beacon transmissions can be observed by chains comprised of linear arrays of receivers. Currently, such chains are located on nearly every continent of the earth, including Asia (Taiwan, Japan), Australia, South America (Argentina), North America (United States, Canada), Europe (United Kingdom, Scandinavia, Russia), and Antarctica for observations of the TRANSIT beacons (Bernhardt, et al., 1998). For tomographic measurements of the ionosphere using the COSMIC TBB, similar linear arrays will have to be set up and aligned with the orbit of the COSMIC satellites and the receivers will have to be tuned to the TBB frequencies instead of the TRANSIT frequencies. TRANSIT radiates at 80 parts per million (ppm) below both 150 and $400 \mathrm{MHz}$. COSMIC will radiate on geodetic frequencies which are 80 ppm above 150 and $400 \mathrm{MHz}$.

A diagram of the radio tomography observing scheme is illustrated in Fig. 4. Each chain of receivers forms a network for accumulating the TEC data from an overflight of the radio beacon. The data are collected and processed using computerized ionospheric tomography (CIT) to produce images of the electron density in the ionosphere. A description of the algorithms is beyond the scope of this paper, but details are available in the literature (Bernhardt et al., 1998). Since TEC measurements used in the 2D reconstructions are based on vertical or oblique propagation paths, the reconstruction suffers from missing information in directions near horizontal. To account for this, the reconstruction algorithms are constrained to relatively lower degrees of freedom in the vertical direction by using limited basis functions or $a$ priori profile measurements.

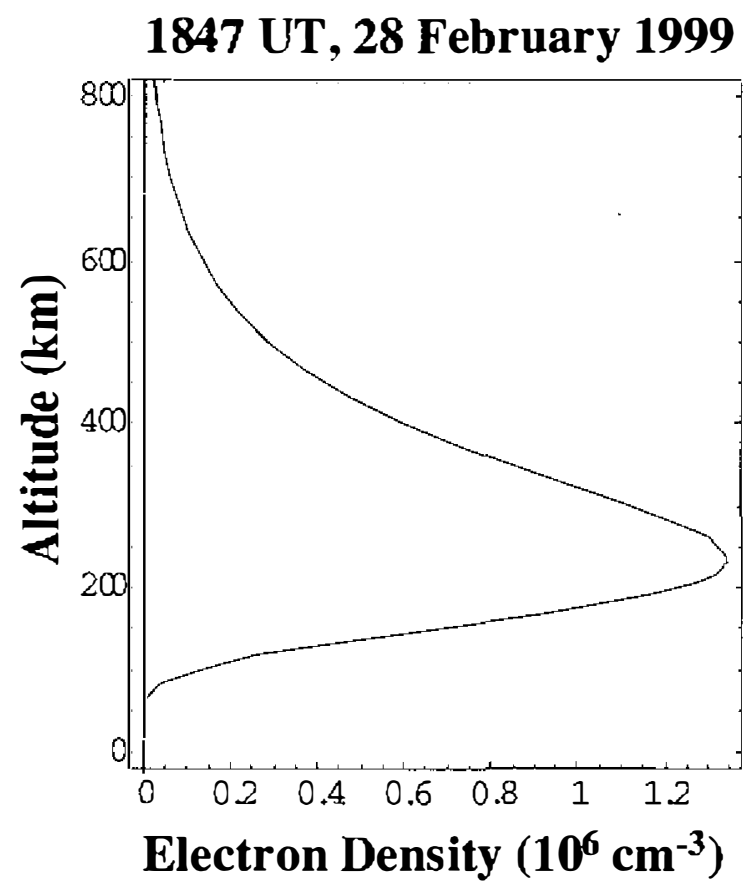

Fig. 4. Electron density profile obtained from TEC data shown in Fig. 3. 
Studies of the high latitude ionosphere by the University of Wales at Aberystwyth are presented for a sample of the results from CIT. Experimental campaigns have been carried out using the Navy Navigation Satellite System (NNSS). The work has been concerned with application of the method to the study of the geophysical processes that control the highlatitude ionosphere that are vital to the understanding of solar-terrestrial relationships. An example of a spatial image of a daytime mid-winter ionosphere is shown in Fig. 5, obtained from observations made at the four stations in the UK (Pryse et al., 1995). The wave train of a traveling ionospheric disturbance can be identified and superposed on the latitudinal gradient of the ionization which can be seen at the layer peak. The inclination of phase fronts indicates that the motion of the associated atmospheric gravity wave was to the south, while the image shows that the latitudinal component of the wavelength of this medium-scale Traveling Ionospheric Disturbance (TID) was about 3 degrees.

Each COSMIC satellite has both a TBB, which is used to measure vertical and oblique TEC, and a GPS receiver used to measure horizontal TEC. As described by Bernhardt et al. (1998), data from these two instruments can be combined to yield higher accuracy images of the ionosphere than can be obtained with each instrument separately. One technique uses GPS occultation measurements to provide electron density profiles that are used as initial conditions for radio-beacon computerized tomography. Another method simultaneously uses both the GPS and TBB measurements of TEC for tomographic reconstructions.

The Tri-Band Beacon experiment on the COSMIC satellite constellation uses a proven

\section{Tri-Band Beacon Observations}

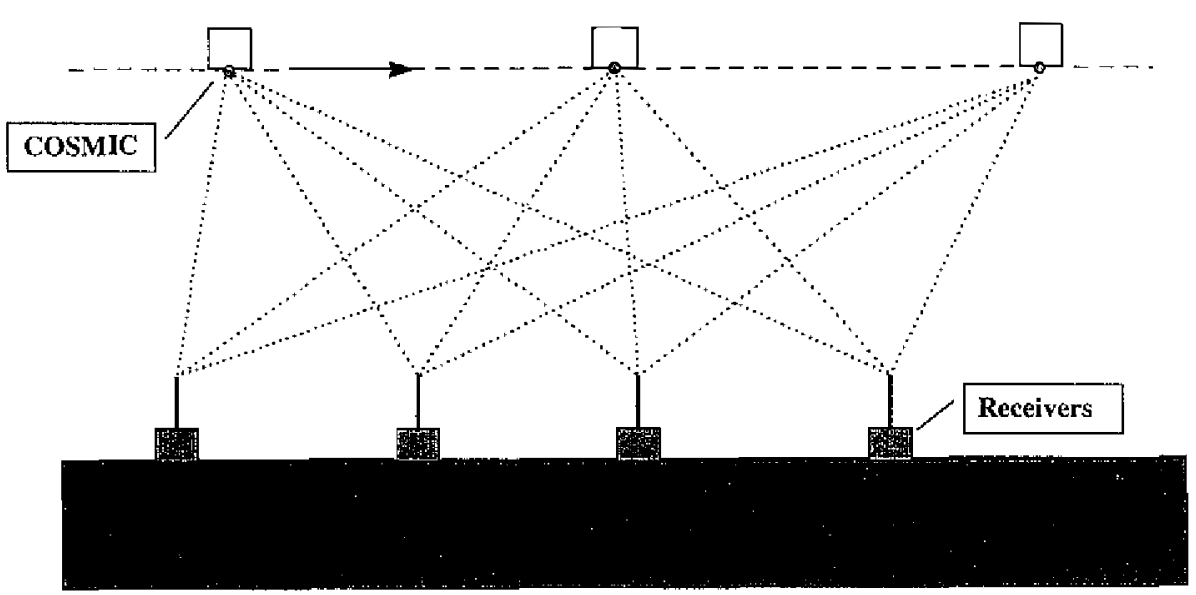

Fig. 5. Ionospheric tomography geometry using the Tri-Band Beacon in low earth orbit on COSMIC. Ground receivers record the integrated electron density along straight line propagation paths. The mesh of intersecting paths permits the reconstruction of F-region ionospheric densities to produce a two-dimensional image. 
technology to produce high resolution images of electron densities in the ionosphere. Because the Tri-Band beacon will be on each of the nine COSMIC satellites, the ionospheric coverage will be greatly expanded in time and space over the current CIT systems which use the US Navy (NNSS) and Russian (CICADA) satellites. These multiple beacons will be used to explore several modes of ionospheric studies. Large-scale natural fluctuations in the ionosphere such as the TID illustrated in Fig. 6 will be imaged on a global basis. These fluctuations can be amplified by electrodynamic processes that convert them into strong irregularities aligned with the magnetic field of the earth (Kelley, 1989). CIT with the Tri-Band Beacon can be use to provide maps of these irregularities with horizontal resolution of about $10 \mathrm{~km}$. The TBB is the only instrument on COSMIC with this resolution. The ground-based receivers monitor amplitude and phase fluctuations of each of the three transmitted frequencies. Each frequency has a corresponding Fresnel zone scale size. Consequently, the effects of ionospheric irregularities that produce radio scintillations can be simultaneously observed for three Fresnel-zone scale-sizes.

The complementary nature of the instruments on COSMIC will be fully exploited. The TBB also yields a means for cross-calibration with the EUV sensor (TIP) on COSMIC. The TIP overflight of ionospheric structures should provide information similar to that obtained from CIT receivers. Occultation of the earth's ionosphere between GPS transmitters and GPS receivers on COSMIC will provide measurements of TEC along horizontal paths that are not

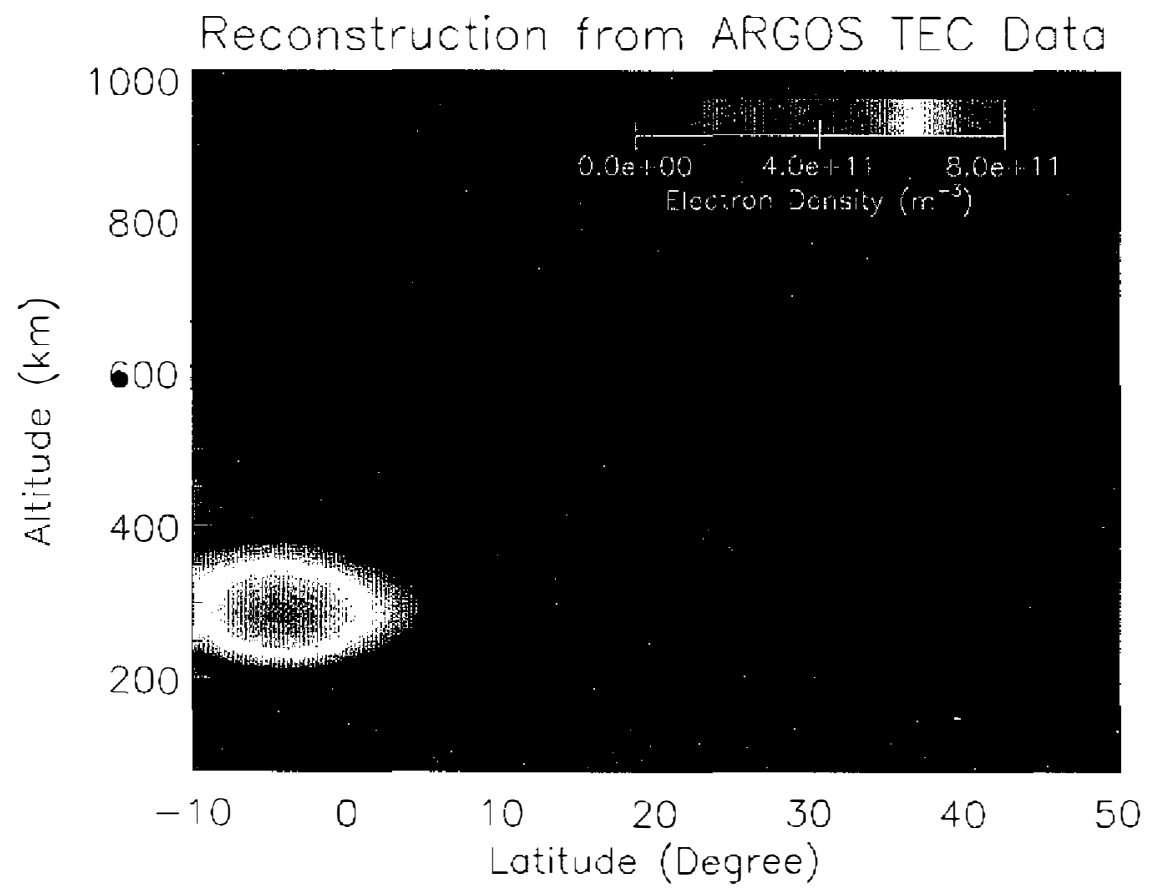

Fig. 6. Tomographic image showing a traveling ionospheric listurbance (TID) over the Caribbean. The data were obtained on 17 September 1999 at -650 UT using the ARGOS dual-frequency beacon. 
provided by the oblique and vertical paths between the TBB and ground receivers. Under conditions where the GPS occultations coincide with TBB overflights of ground receivers, the two data sets will be combined to yield improved images of the ionosphere (Bernhardt et al., 1998).

\section{RADIO WAVE SCINTILLATION MONITORING}

Scintillations are a major problem for radio systems using satellite to ground links near the magnetic equator and at the high-latitudes that include the polar cap and auroral regions. VHF communication links can be affected by scintillations at all times during the solar cycle, with the severity peaking near the period of solar maximum (Basu, Mackenzie, and Basu 1988). At UHF and the GPS frequencies at L-band (1.2 GHz and 1.6 GHz), strong scintillations are encountered during the solar maximum period, especially near the magnetic equator and in the Asian sector (Fang and Liu, 1983 and 1984). At GPS frequencies, scintillation greater than $20 \mathrm{~dB}$ may be observed near the crests of the equatorial anomalies at $15^{\circ} \mathrm{N}$ and $15^{\circ} \mathrm{S}$ magnetic latitudes. Scintillations during a solar maximum may cause the loss of phase lock in GPS receivers (Groves et al., 1997).

Amplitude and phase scintillations are often associated with large gradients in TEC. At high latitudes, these gradients are associated with enhanced ionization density as in polar cap patches or auroral blobs. At low latitudes, these gradients are observed in plasma bubbles or "depletions" of plasma density (Fig. 7). The Tri-Band Beacon experiment on COSMIC will provide simultaneous measurements of TEC along with phase and amplitude scintillations at 150, 400 and $1067 \mathrm{MHz}$. To the first order approximation, the Tri-band Beacon will allow the determination of the frequency dependence of scintillation in weak and strongly turbulent structures near the magnetic equator. With simultaneous TEC determination, the distribution of scintillation causing irregularities with respect to the TEC structures will be determined. As represented by Fig. 7, at the VHF frequency of $137 \mathrm{MHz}$, scintillations are observed continuously over the entire TEC reduction. On the other hand, at the higher L-band frequency, scintillations are more discrete and occur near steep TEC gradients.

Most of the tomographic analysis of ionospheric irregularities is restricted to TEC data for providing images of large scale structures in the ionosphere. This type of tomography was described in the previous section. Mapping ionospheric regions that are responsible for scintillations has been proposed as either (1) diffraction radiotomography or (2) statistical radio tomography (Kunitsyn et al., 1994). The difference between these techniques is that the full diffraction pattern of the wave is measured in (1) so that each individual irregularity can be reconstructed and in (2) so that tomographic inversion is used to provide the statistical characteristics of the region. The latter technique will be investigated with the COSMIC TBB to provide images of ionospheric "roughness".

\section{TROPOSPHERIC MONITORING WITH THE COSMIC TBB IN LOW EARTH ORBIT}

With the emergence of the GPS technology for providing high geodetic quality position- 


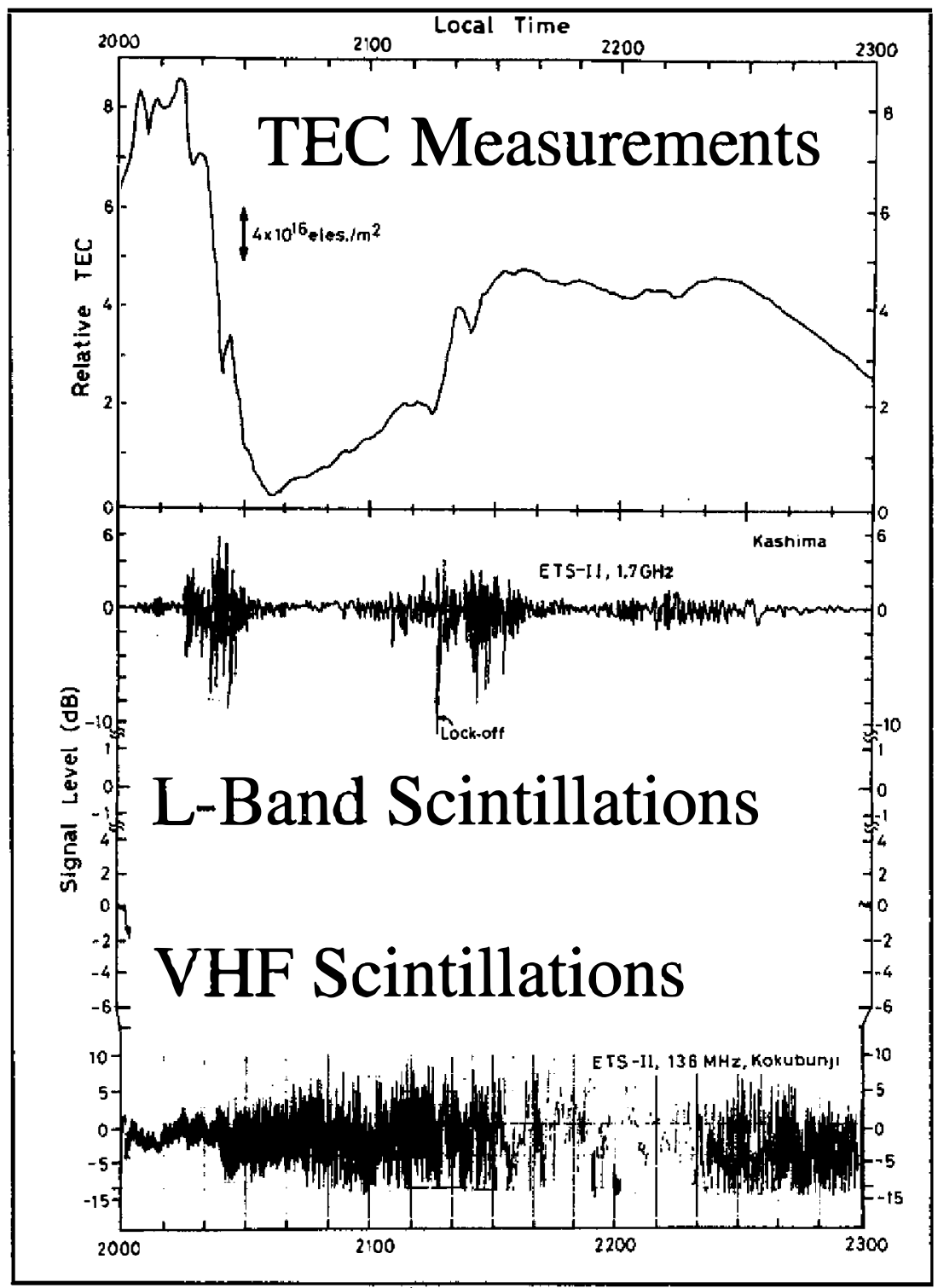

Fig. 7. Scintillations observed in Japan correlated with fluctuations in total elecron content (TEC). The VHF scintillations are $10 \mathrm{~dB}$ stronger than those at L-Band. (Adapted from Davies [1990].)

ing and time clock synchronization, the opportunity for monitoring other geophysical parameters affecting the GPS signals can be exploited. Water vapor in the Earth's lower atmosphere (or troposphere) is one such parameter. The COSMIC constellation has the potential for similar monitoring using the low-Earth orbiting (LEO) TBB in the VHF, UHF and L-bands. If the water vapor measurements are performed with sufficient precision, they will be an inexpen- 
sive alternative to water vapor estimations from radiometers. Additionally, the geometry of the measurements will be well suited to the use of tomographic techniques to produce twodimensional images of the water vapor along the orbital plane. Just as tomography has been used to image the ionosphere (see Section V.), a similar approach can be employed to provide two-dimensional maps of water vapor in the troposphere.

The refraction of radio waves by the neutral atmosphere (dry gases and water vapor) is independent of frequency, producing delays in the signal from the satellite to the receiving station. The troposphere is the lower part of the Earth's atmosphere (up to about $20 \mathrm{~km}$ ) and contains the bulk of the neutral atmosphere contributing to signal delay. Tropospheric delay has a hydrostatic component (dry gases) and a wet component (water vapor). The hydrostatic component is larger but far less variable and can be predicted from surface pressure measurements. The wet delay along a particular radio signal path is essentially a measure of the total amount of water vapor through which the signal passes.

Water vapor varies with geographic region and local weather conditions, contributing from 0 to a few $\mathrm{cm}$ of vertical radio delay in the dry polar regions and from 20 to $40 \mathrm{~cm}$ of vertical delay in wet equatorial regions. Slant paths at very low elevation angles pass through significantly larger amounts of water vapor than vertical paths. The slant paths can have wet delays that are a factor of five larger than that of a vertical paths. So at a site with $20 \mathrm{~cm}$ of vertical wet delay, slant paths could be delayed by $1 \mathrm{~m}$ or so due to water vapor.

GPS ground stations are currently being used by NOAA to monitor the troposphere in near real-time (Gutman et al., 1994; Bevis al., 1996; Rocken, VanHove and Ware, 1997). The zenith wet delay over each station in a network is estimated using geodetic processing techniques. At least one baseline in the network must be longer than approximately $500 \mathrm{~km}$, otherwise a water vapor radiometer is required at one site in the network for accurate bias determination. Double differencing of the Doppler data is used to reduce the effects of satellite orbit uncertainties and to remove errors due to satellite and receiver clock variability, as well as selective availability. Ionospheric effects are removed using equation (3) or by an improved dual-frequency correction algorithm which includes a model for higher order ionospheric terms (Davies, 1990). The hydrostatic delay term of the troposphere is modeled using surface pressure measurements. Since the path length term is well characterized, the only remaining terms are the tropospheric delays due to water vapor (or wet delay), multipath, system noise, and residual errors in the other terms. The zenith wet delay at each station is estimated from the relative double differenced measurements using either a least squares inversion or a Kalman filtering approach. Using these techniques, zenith wet delay is obtained with an accuracy and precision better than $1 \mathrm{~cm}$.

A similar differencing technique can be applied to Doppler data received from a lowEarth orbiting (LEO) Doppler beacon, such as the TBB frequencies of 150, 400, and 1067 $\mathrm{MHz}$ on the COSMIC satellites. By differencing the data recorded at two ground sites and by removing the other terms, the differential tropospheric wet delay is obtained. This can be repeated for all the sites in the network. Absolute wet delay is not required, as long as accurate measurements of the change in differential wet delay are made across the network. Once the differential wet delay as a function of elevation has been inferred for each station, the strong elevation dependence and overlapping sampling from multiple stations can be used to resolve 
the absolute wet delay. This method is analogous to the Leitinger technique for estimating total electron content (Leitinger, Schmidt and Tauiainen, 1975).

Clock errors are a potential source of uncertainty in the water vapor measurements. The drift of the oscillator on the TBB is minimized using an oven for stabilization. Differencing the Doppler data recorded at two ground sites accounts for most of the orbit error and all of the satellite clock error. Receiver clock bias is not important but receiver clock drift is. For the error in wet delay to be less than $1 \mathrm{~cm}$ over 20 minutes, the receiver clock drift must be determined to better than $3 \times 10^{-14}$ seconds per second or be common to all receivers in the network.

For station separations less than $100 \mathrm{~km}$, less than $0.5 \%$ of the orbit error remains after differencing. Since orbits are now determined to better than $10 \mathrm{~cm}$ using GPS or $100 \mathrm{~cm}$ using the TBB transmissions (see next section), the satellite position error will contribute less than a $\mathrm{mm}$ to wet delay observations. The delay error in relating the receiver clocks to a common clock using GPS is estimated to be $7 \mathrm{~mm}$ over 20 minutes. The ionospheric delay, including the higher order terms, is removed using a combination of the three widely separated frequencies. The precision of this correction is driven primarily by the lower frequencies (150 and $400 \mathrm{MHz}$ ) and is estimated to be less than $7 \mathrm{~mm}$. If accurate surface pressure measurements (1 mbars or better) are available at the receiving sites, then the residual hydrostatic delay of the troposphere contributes less than $3 \mathrm{~mm}$ of error. System noise on the differential $1067 \mathrm{MHz}$ signal is estimated to be $8 \mathrm{~mm}$. Therefore, the total error budget for differential wet delay is 1$2 \mathrm{~cm}$. In the tropics, $1-2 \mathrm{~cm}$ gives about $1 \%$ to $5 \%$ of error in estimating wet delay. The error budget given in Table 2 is a conservative estimate. It is likely that receiver clock drift, the ionospheric correction term and system noise will all be smaller than these estimates. Further, tomographic methods should tend to average down many of the error terms.

Once the differential wet delay is obtained between any two ground receivers, the absolute wet delay can be estimated by several methods. The first method is to employ a radiometer at one site and to infer the wet delay at the second site. It is not necessary that the radiometer be calibrated absolutely, only that the wet delay as a function of elevation be precise. The second method is to combine the LEO beacon Doppler data with other data sources such as ground GPS or LEO GPS receivers, and to infer absolute delay. A third method is to apply simple model constraints to the multi-station data in order to determine the absolute bias of each data source using the strength of the overlapping elevation dependencies. This is analogous to the Leitinger technique for determining absolute TEC from a chain of TRANSIT receivers. This method can yield the absolute wet delay along the line-of-sight path from the LEO satellite to each receiving station in the network.

Using the differential wet delay, tomographic techniques will be employed to reconstruct two-dimensional images of the water vapor density in the troposphere. The geometry of the tropospheric data, collected from a chain of receivers along the satellite orbit plane, is well suited for tomography. All data are collected during a single pass of the satellite over the region (approximately $20 \mathrm{~min}$ ), so the assumption of a static medium is a reasonable one. Receiver spacing on the order of 5-6 km and satellite tracking to $10 \mathrm{o}$ elevation will produce a sufficient number of intersecting paths to reconstruct water vapor density profiles from 1-40 $\mathrm{km}$ altitudes along the entire chain. Many of the profiles along the chain would reach altitudes 
Table 2. Error Budget in Determination of Water Vapor Delay.

\begin{tabular}{|c|c|}
\hline Parameter & RMS Uncertainty \\
\hline Satellite Position & $1 \mathrm{~mm}$ \\
\hline Ground Position & $5 \mathrm{~mm}$ \\
\hline Satellite Clock Drift & $0 \mathrm{~mm}$ \\
\hline Receiver Clock Drift & $7 \mathrm{~mm}$ \\
\hline Ionospheric Residual Delay & $0 \mathbf{m m}$ \\
\hline Ionospheric Correction Noise & $7 \mathrm{~mm}$ \\
\hline Hydrostatic Residual Delay & $3 \mathrm{~mm}$ \\
\hline System Noise (1067 MHz) & $8 \mathrm{~mm}$ \\
\hline Differential Wet Delay Error & $14 \mathrm{~mm}$ \\
\hline
\end{tabular}

as low as $500 \mathrm{~m}$. With larger station separations or with higher minimum elevation angles, the minimum reconstruction altitude increases. Unlike ionospheric tomography from groundbased receivers, in which the altitude of the ray crossings and curvature of the Earth limit the vertical resolution of the data, nearly horizontal ray crossings occur at the lower altitudes in tropospheric tomography. Tomography at low altitudes (approx. 1 to $3 \mathrm{~km}$ ) yields better vertical resolution than horizontal resolution.

Thus, techniques used to monitor the troposphere from GPS signals can be adapted to LEO Doppler beacons to observe differential wet delay above a network of ground receivers. The strong elevation angle dependence of the observations allows measurement of the absolute wet delay at each station. The rapid over flight of the satellite and the geometry of crossing rays observed from a chain of receivers along the satellite orbital plane allow tomographic techniques to be used to reconstruct water vapor profiles.

\section{HIGH RESOLUTION ORBIT DETERMINATION FOR MEASUREMENT OF SATELLITE DRAG}

Since 1964, empirical models of the upper atmosphere have been developed from satellite drag data (Jacchia, 1970; Jacchia, 1971). These models include parameterized influences of solar flux with a solar cycle and daily component, geomagnetic activity, local time, day of year, latitude and longitude. Factors of two or more variations in neutral density with time scales of hours can be observed in the neutral atmosphere. Tracking of satellites is accomplished by a number of techniques including (1) radar ranging, (2) onboard GPS receiver, or 
(3) onboard Doppler beacon transmitter. The COSMIC constellation can employ each of these three techniques for orbit determination but use of the TBB is described here.

Doppler measurements from ground based receivers of the TBB signals can provide precise orbits of each COSMIC satellite. The stability of the TBB is specified to be 1 part in $10^{12}$ which yields a displacement error of $20 \mathrm{~cm}$ in position (Newton, 1976; Gu and Brunner, 1990, Hartmann and Leitinger, 1984). Other system errors for the COSMIC satellites, like unmodeled forces, residual fraction and antenna pattern are in the 1 meter range.

Atmospheric drag is the predominant natural force that affects the COSMIC orbits, especially when the satellites are near their injection altitude of $400 \mathrm{~km}$ (Rocken et al., 1999). The orbit data obtained using COSMIC TBB Doppler position technique can be analyzed to yield the drag acceleration. Orbital drag accelerations for each COSMIC satellite are related to the satellite structure and the neutral density by

$$
a_{D}=1 / 2\left(C_{D} A / M\right) \rho V^{2},
$$

where $a_{D}$ is the drag acceleration, $\rho$, the atmospheric total mass density, $A$ the satellite area, $M$ the satellite mass, $C_{D}$ drag coefficient, and $V$ velocity (Marcos et al.,1998). The ballistic coefficient $B=C_{D} A / M$ is assumed to be constant and any variations in the drag acceleration is due to the variations in the neutral density $\rho$. Over the period of 10 days, the acceleration drag can fluctuate by more than $60 \%$. The orbit determination using the TBB for Doppler ranging can provide a scientific data source for a global map of these fluctuations.

\section{SUMMARY}

The Tri-Band Beacon on the COSMIC constellation is a versatile device that can be employed to study the troposphere and the upper atmosphere. Atmospheric applications rely on a frequency stable transmitter that radiates phase coherent signals at VHF, UHF, and L-band. An omni-directional antenna is required to illuminate the earth uniformly at all three frequencies. The transmitted frequencies are oven stabilized to permit extraction of both satellite position from Doppler ranging data and integrated water vapor density from single-frequency phase delay. The satellite position data can be analyzed to yield drag accelerations and estimations of neutral density. Two dimensional images of precipitable water vapor may be obtained using tomographic analysis of column densities of water vapor between a COSMIC satellite orbit and a linear array for ground receivers. Finally, ionospheric parameters can be obtained from phase and amplitude measurements at the three TBB frequencies are recorded with groundbased receivers. The data from these receivers are processed to yield images of ionospheric electron densities and irregularities using single-station profiling or multi-station, tomographic analysis techniques.

The complementary nature of the TBB and GPS receiver on COSMIC will be fully exploited for both ionospheric and tropospheric observations. The TBB provides three frequencies at VHF, UHF and L-Band for transmission to highly stable transmissions to arrays of ground receivers. The GPS receiver records L-band transmissions from the constellation of GPS satellites around the globe. The differential phase measurements using the VHF/UHF pair from the TBB are inherently more accurate than the L-Band GPS measurements because, 
as shown by Equation (2), the phase enhancement is inversely proportional to radiated frequency. The GPS receiver, however, can more easily resolve $2 \pi$ phase ambiguities because of its lower phase resolution and by using group-delay techniques not available with the TBB system. The radio wave ransmissions from the TBB in low-earth-orbit provide scans of ionospheric structures during an over-flight. These scans highlight the horizontal variations in the structures but are less sensitive to vertical structures. The GPS receiver, by contrast, is employed to make limb-scans of the ionosphere to yield vertical structures with poor resolution of horizontal variations. Simultaneous measurements with both instruments eliminate the limitations of each individual technique. Identical comments can be applied to the tropospheric measurements where the TBB should provide horizontal fluctuations while the GPS occultations would provide vertical profiles of water vapor. Both the TBB and GPS receiver systems can obtain independent measurements of satellite position. The TBB technique has been used for twenty years and the GPS receiver is a relatively new addition to satellite-based position measurements. Cross-calibration of the satellite orbit determined using the two instruments will provide validation of both techniques.

Acknowledgments The authors are grateful for discussions with Dr. J.D. Huba. This research was sponsored by the Office of Naval Research and the COSMIC Program Office at the University Corporation for Atmospheric Research.

\section{REFERENCES}

Austen, R. J., S. J. Franke, and C. H. Liu, 1988: Ionospheric imaging using computerized tomography. Radio Sci., 23, 299-307.

Bernhardt, P.A., R. P. McCoy, K. F. Dymond, J. M. Picone, R. R. Meier, F. Kamalabadi, D. M. Cotton, S. Charkrabarti, T. A. Cook, J. S. Vickers, A. W. Stephan, L. Kersley, S. E. Pryse, I. K. Walker, C. N. Mitchell, P. R. Straus, H. Ha, C. Biswas, G. S Bust, G. R. Kronschnabl, and T. D. Raymund, 1998: Two-dimensional mapping of the plasma density in the upper atmosphere with computerized ionospheric tomography (CIT). Physics of Plasmas, 5, 2010-2021.

Bevis, M., S. Businger, T. Herring, C. Rocken, R. Anthes, and R. Ware, 1992: GPS Meteorology: Remote Sensing of Atmospheric Water Vapor Using GPS. J. Geophys. Res, 97, 15787-15801.

Bevis, M., S. Businger, S. Criswell, T. Herring, R. Anthes, C. Rocken, and R. Ware, 1994: GPS Meteorology:Mapping Zenith Wet Delays onto Precipitable Water. J. Appl. Meteorol., 33, 379-386.

Bevis, J., M., P. Fang, Y.Bock, S. Chiswell, S. Businger, C. Rocken, F. Solheim, T. VanHove, R. Ware, S. McClusky, T. A. Herring, and R. W. King, 1996: GPS Meteorology: Direct Estimation of the Absolute Value of Precipitable Water. J. Applied Met., 35, 830-838.

Bust, G. S. J. A. Cook, G. R. Kronschnabl, C. J. Vasicek, and S. B. Ward, 1994: Int., Application of ionospheric tomography to single-site location range estimation. Int. J. Imag. Syst. Tech., 5, 160-168.

Davies, K., 1990: Ionospheric Radio, IEE Electromagnetic Waves Series 31, Peter Peregrinus 
Ltd., Exeter, England.

Fang, D. J., and C. H. Liu, 1984: Statistical Characterizations of Equatorial Scintillation in the Asian Region. Radio Sci., 19, 345-358.

Fang, D. J., and C. H. Liu, 1983: A morphological-study of gigahertz equatorial scintillations in the Asian region. Radio Sci., 18, 241-252.

Fehmers, G., 1996: Tomography of the Ionosphere, Thesis: Eindhoven University of Technology.

Foster, J. C., M. J. Buonsanto, J. M. Holt, J. A. Klobuchar, P. Fougere, W. Pakula, T. D. Raymund, V. . Kunitsyn, E. S. Andeeva, E. D. Tereshchenko, and B. Z. Khudukon, 1994: Russian-American tomography experiment. Int. J. Imag. Syst. Tech., 5, 148-159.

Groves, K. M., S. Basu, E. J. Weber, M. Smitham, H. Kuenzler, C. D. Valladares, R. Sheehan, E. MacKenzie, J. A. Secan, P. Ning, W. J. McHeill, D. W. Moonan, and M. J. Kendra, 1997: Equatorial scintillation and systems support. Radio Sci., 32, 2047-2064.

$\mathrm{Gu}$, M., and F. Brunner, 1990: Theory of the two frequency dispersive range correction. Manuscripta Geodaetica, 15, 357-361, .

Gutman, S., R. Chadwick, D. Wolfe, A. Simon, T. Van Hove, and C. Rocken, 1994: Toward an Operational Water Vapor Remote Sensing System Using GPS, FSL Forum, September.

Hartmann G. K., Leitinger R., 1984: Range errors due to ionospheric and tropospheric effects for signal frequencies above $100 \mathrm{MHz}$. Bull. Geod., 58, 109-136.

Huang C. R., C. H. Liu, K. C. Yeh, K. H. Lin, W. H. Tsai, H. C. Yeh, J. Y. Liu, 1999: A study of tomographically reconstructed ionospheric images during a solar eclipse. J. Geophys. Res, 104, 79-94.

Jacchia, L. G., 1970: Revised Static Models of the Thermosphere and Exosphere with Empirical Temperature Profiles, SAO Report No. 313.

Jacchia, L. G., 1971: Revised Static Models of the Thermosphere and Exosphere with Empirical Temperature Profiles, SAO Report No. 332.

Kelley, M. C., 1989: The Earth's Ionosphere, pp. 261-310, Academic Press.

Kersley, L., S. E. Pryse, I. K. Walker, J. A. T. Heaton, C. N. Mitchell, M. J. Williams, and C. A. Willson, 1997: Imaging of electron density troughs by tomographic techniques. Radio Sci., 32, 1607-1621.

Kunitsyn, V. E., E. S. Andreeva, E. D. Tereshchenko, B. Z. Khudukon, and T. Nygren, 1994: Investigations of the ionosphere by satellite radiotomography. Int. J. Imag. Syst. Tech., 5, $112-127$.

Kunitsyn, V. E., S. A. Andreeva, E. D. Tereshchenko, B. Z. Khudukon, T. Nygren, 1994: Investigations of the ionosphere by satellite radio tomography. Int. J. Imaging Systems and Technology, 5, 112-127.

Leitinger, R., G. Schmidt, and A. Tauiainen, 1975: An evaluation method combining the differential Doppler measurements from two stations that enables the calculation of electron content of the ionosphere. J. Geophys. Res., 41, 201-213.

Marcos, F. A., M. J. Kendra, J. M. Griffin, J. N. Bass, D. R. Larson, and J. J. F. Liu, 1998: Precision low earth orbit determination using atmospheric density calibration, AAS 96-631, pp501-527, Astronautics 1997, Part I, Univelt Inc., San Diego. 
Newton, R. R., 1976: The Navy Navigation Satellite System, Space Research VII, pp. 735763, North-Holland.

Pryse, S. E., C. N. Mitchell, A. A. T. Heaton, and L. Kersely, 1995: Traveling ionospheric disturbances imaged by tomographic techniques. Ann. Geophysicae, 13, 1325-1330.

Raymund, T. D., R. J. Austen, S. J. Franke, C. H. Liu, J. A. Klobuchar, and J. Stalker, 1990: Application of computerized tomography to the investigation of ionospheric structures. Radio Sci. 25, 771-789.

Rishbeth, H. and O.K Garriott, Introduction to Ionospheric Physics, pp. 126-159, Academic Press, New York 1969.

Rocken, C., T. Van Hove, 1997: Randolph Ware, Near real-time GPS sensing of atmospheric water vapor. J. Geophys. Res., 24, 3221-3224.

Rocken, C., T. VanHove. R. Ware, 1997: Near real-time GPS sensing of atmospheric water vapor. Geophys. Res. Let., 24, 3221-3224.

Rocken, C., Y.-H. Kuo, W. Schreiner, K. Brock, D. Hunt, S. Sokolovskiy, 1999: COSMIC System Description. TAO, 11, 21-52 .

S. Basu, E. Mackenzie, and Su. Basu, 1988: Ionospheric constraints on VHF/UHF communication links during solar maximum and minimum periods. Radio Sci., 23, 363.

Sayenko, Y. S., I. I. Shagimuratove, A. N. Namgaladze, G. A. Yakimova, N. S. Natsvalian, O. V. Biriukov, and V. I. Kuzin, 1991: Restoration of the ionospheric electron density distribution from tomographic processing of satellite radio signals. Geomagnetism Aeronomy, 31, 558-561.

Yeh, H. C., S. J. Franke, K. C. Yeh, C. H. Liu, T. D. Raymund, H. H. Chen, A. V. Izotov, J. Y. Liu, J. Wu, K. H. Lin, and S. W. Chen, 1994: Low-latitude ionospheric tomography network along Taiwan meridian, Low-Latitude Ionospheric Physics, pp. 265-304, COSPAR Colloquia Series, Vol. 7, 295-303, Elsevier Science, Inc., Oxford.

Yeh, K. C., and T. D. Raymund, 1991: Limitations of ionospheric imaging by tomography. Radio Sci., 20, 1361-1380. 